\title{
Fluoride prevents caries among adults of all ages
}

\author{
Is fluoride effective in preventing coronal caries in all adults and in older \\ adults (40+ years) and in preventing root caries in the older group?
}

How effective are the different fluoride delivery modes in preventing caries?

\author{
Griffin SO, Regnier E, Griffin PM, Huntley V. \\ Effectiveness of fluoride in preventing caries in adults. J Dent Res \\ 2007; 86:410-415
}

Data sources Medline, Embase and the Cochrane Central Register of
Controlled Trials were searched. References of each retrieved article were
also searched for relevant articles. The US Food and Drug Administration,
American Dental Association and manufacturers of topical fluoride prod-
ucts were contacted to identify unpublished trials.
Study selection For inclusion, the study had to be published in the
English language, was of duration 1 year or longer, and examined the
association between fluoride and caries in intact human teeth in study
populations that included adults. To examine the effectiveness of self- or
clinically applied fluoride, only longitudinal studies with random assign-
ment of participants or of split-mouth design were included. For studies
examining the effectiveness of water fluoridation, cross-sectional studies
were included if their participants lived most of their lives in a fluoridated
or a nonfluoridated community. Data extraction and synthesis Screening of eligible studies and data extraction were conducted independently and in duplicate. A random-effects model was used to estimate the effect-size of fluoride (absolute difference in annual caries increment or relative risk ratio) for all adults aged $20+$ years and for adults aged over 40 years. Root caries increment was also estimated for adults aged over 40 years.

Results Twenty studies involving 13551 participants were included in the final body of evidence. Eleven studies examined the effectiveness of self- or clinically applied fluoride. Of these studies, 10 were randomised controlled trials, and one was a controlled trial (split-mouth) that did not specify whether the treatment had been randomly assigned. Nine studies examined the effectiveness of water fluoridation; all were cross-sectioned studies except one which was a prospective cohort trial. Of the studies published after or during 1980, any fluoride (self- and clinically applied or water fluoridation) annually averted 0.29 [95\% confidence intervals (Cl), 0.16-0.42] carious coronal and $0.22(95 \% \mathrm{Cl}, 0.08-0.37)$ carious root surfaces. The prevented fraction for water fluoridation was $27.2 \%$ (95\% Cl, 19.4-34.3\%).

Conclusions These findings suggest that fluoride is effective in preventing caries in adults of all ages.

Address for correspondence: SO Griffin, Centers for Disease Control and Prevention/ Division of Oral Health, 4770 Buford Highway, MSF10, Chamblee GA 30341, USA. E-mail: sig1@cdc.gov

\section{Commentary}

The effectiveness of different fluoride delivery systems has been evaluated primarily in children and adolescents and the results have, in most instances, been extrapolated to adults. Recent research ${ }^{1}$ suggests that adults are keeping their teeth longer and are at similar or higher risk for dental caries than are children.

The objective of this systematic review was to examine the effectiveness of self- and clinically applied fluoride and water fluoridation among adults. The inclusion and exclusion criteria were explicit. The search strategy, however, appeared to be too simple. It was not clear whether a controlled vocabulary had been used in searching electronic databases. Restriction to English-language studies may also have had an effect on the findings.

It is interesting to compare the findings of this review to the systematic review conducted by McDonagh et al. (York review) ${ }^{2}$ In the current review, cross-sectional studies were included to examine the effectiveness of water fluoridation, but cross-sectional studies were excluded from the York review ${ }^{2}$ as it was not possible to tell whether the observed differences in caries experience had always existed between these populations or whether they were the result of differing levels of water fluoride content between the study areas. Out of the 26 included studies in the York review, ${ }^{2}$ that by Pot et al. ${ }^{3}$ was the only one whose participants were adults. Because the study by Pot and colleagues was published in Dutch, it was excluded from this recent systematic review. In other words, the studies included in these two systematic reviews were completely different!

The findings of the York review ${ }^{2}$ showed that there was a median change of $14.6 \%$ in the proportion of caries-free children, roughly equivalent to a prevented fraction of $40 \% .{ }^{4}$ In this recent systematic review, the prevented fraction for water fluoridation was only $27 \%$. Such a difference is not unexpected because of the pre-eruptive effects of fluoridated water.

The authors compared their results with those of Cochrane reviews on fluoride mouthrinse and fluoride toothpaste in children and adolescents: the findings were surprisingly similar. The authors, however, did not compare their findings with the systematic reviews by Twetman et al. ${ }^{5,6}$ in which both adults and children were considered. Twetman and colleagues ${ }^{5}$ found limited evidence that fluoride mouthrinse prevented root caries in older adults. Regarding fluoride toothpaste, all the studies of adult participants identified by Twetman et al. ${ }^{6}$ were dealing with materials or additional active ingredients other than fluoride. Therefore, none of these studies fulfilled the inclusion criteria and they were excluded.

The retention of a functional and natural dentition for life is now a realistic goal for most individuals. In view of the findings in the above systematic reviews, further well-designed studies are needed to elucidate the effectiveness of fluoride among adults. 


\section{Practice point}

Fluoride prevents caries in adults of all ages.

\section{Albert Yeung}

Department of Public Health, National Health Service Lanarkshire, Hamilton, Lanarkshire, Scotland, UK

1. Griffin SO, Griffin PM, Swann JL, Zlobin N. New coronal caries in older adults: implications for prevention. J Dent Res 2005; 84:715-720.

2. McDonagh $M$, Whiting $P$, Bradley $M$, et al. A systematic review of public water fluoridation. York: National Health Service Centre for Reviews and Dissemination, University of York; 2000.
3. Pot T, Purdell-Lewis DJ, Groeneveld A. The influence of 17 years of water fluoridation upon the dentition of adults. Ned T Tandheelk 1974; 81:5-12.

4. Worthington $\mathrm{H}$, Clarkson I. The evidence base for topical fluorides. Commun Dent Health 2003; 20:74-76.

5. Twetman S, Petersson LG, Axelsson S, et al. Caries-preventive effect of sodium fluoride mouthrinses: a systematic review of controlled clinical trials. Acta Odontol Scand $2004 ; 62: 223-230$.

6. Twetman S, Axelsson S, Dahlgren $\mathrm{H}$, et al. Caries-preventive effect of fluoride toothpaste: a systematic review. Acta Odontol Scand 2003; 61:347-355.

Evidence-Based Dentistry (2007) 8, 5-6. doi:10.1038/sj.ebd.6400506

\section{Key to evidence graphic used in the Evidence-based Dentistry Journal}

The graphic is based on the Centre for Evidence-based Medicine levels of Evidence tables www.cebm.net/levels_of_evidence.asp (see Evidence-based Dentistry 2003;4: p 17-18)

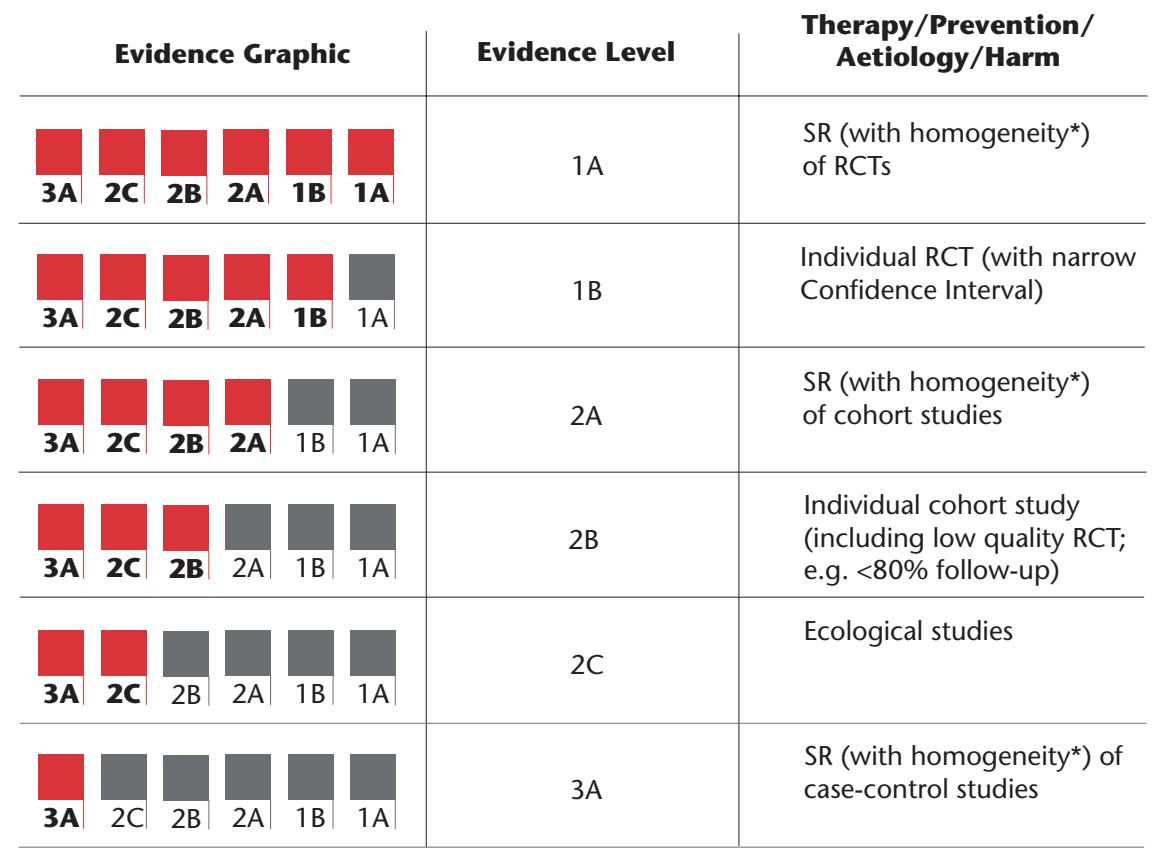

* By homogeneity we mean a systematic review that is free of worrisome variations (heterogeneity) in the directions and degrees of results between individual studies. Not all systematic reviews with statistically significant heterogeneity need be worrisome, and not all worrisome heterogeneity need be statistically significant. 